\title{
A Survey on Fatigue Detection of Workers Using Machine Learning
}

\author{
Nisha Yadav, JSS Academy of Technical Education, Noida, India \\ Kakoli Banerjee, JSS Academy of Technical Education, Noida, India \\ Vikram Bali, JSS Academy of Technical Education, Noida, India
}

\begin{abstract}
In the software industry, where the quality of the output is based on human performance, fatigue can be a reason for performance degradation. Fatigue not only degrades quality, but is also a health risk factor. Sleep disorders, depression, and stress are all results of fatigue which can contribute to fatal problems. This article presents a comparative study of different techniques which can be used for detecting fatigue of programmers and data miners who spent lots of time in front of a computer screen. Machine learning can used for worker fatigue detection also, but there are some factors which are specific for software workers. One of such factors is screen illumination. Screen illumination is the light of the computer screen or laptop screen that is casted on the workers face and makes it difficult for the machine learning algorithm to extract the facial features. This article presents a comparative study of the techniques which can be used for general fatigue detection and identifies the best techniques.
\end{abstract}

\section{KEYWORDS}

Drowsiness, Estimation, Extraction, Face Detection

\section{INTRODUCTION}

Different research shows the effect of vehicle driver fatigue. But fatigue has an effect on workers at different kinds of industry. Work fatigue can affect people working on a shift basis, people affected by high noise, pollution, chemicals, and pressure. The current study focuses on the tiredness of software developers and data miners who spend more time in front of the computer or laptop screen.

As the software industry moves towards a more human-centred environment, software developer health care becomes a very important factor where software developers suffer from depression, anxiety, sleep disorders, etc., it becomes important for employees to ensure that software developers do not suffer from signs or effects of fatigue.

Fatigue also has many long-term effects that affect output productivity and quality. Some of the long-term effects of fatigue affecting the software industry are: inability to stay awake, forgetfulness, error of judgment, inability of complex planning, etc. This results in work errors and can also affect the health of workers. Workers who work for longer hours or additional time may also be in danger, as shown by an article in the NSC's Safety + Health magazine. A progress report by the National Institute for Occupational Safety and Health (NIOSH) found that workers in progress occupations have to rest about 7 hours a day compared to all other groups. (source: https://ehsdailyadvisor.blr. 
com/2017/06/fatigued-workers-hazard-company/).It should be detected and resolved before fatigue becomes a problem and lowers productivity and becomes fatal.

In this paper, as we focus on software developers 'fatigue detection, there are some factors that need to be addressed. As we focus on facial features and especially eye expressions for fatigue detection, the need to capture facial images is required. Once the algorithm captures the images of the face, different features can be extracted and fatigue can be detected. But the most important factor, facing by the algorithm while extracting data from the image captured on the computer screen via a webcam or an inbuilt camera is illumination. In this review paper, a comparison of different techniques is used to detect facial fatigue. The best technique that can take care of the onscreen illumination problem is also identified along with the comparison.

Following are the figures given below that shows the fatigue cycle during the tasks Figure 1 Shows the fatigue related risk and protection against the fatigue with reviews. This figure also explains the scope of risks according to the work type with some implementations.

Figure 2 shows the fatigue of workers occurred during the work. There are some tips for workers to decreasing the fatigue level as shown in the figure. Also, the impacts of fatigue have been explained in the figure.

\section{RELATED WORK}

Qi, Li, Wang, Zhang, Xing, Gao, and Zhang in 2018 proposed a new methodology to recognize expressions based on cognitive and mapped binary patterns. First approach depends on the LBP operator to remove facial highlights, second was the foundation of the pseudo 3-D model to segment the facial region into six featured parts. Finally, they played out a similar investigation on the extension of Cohn-kanade $(\mathrm{CK}+)$ outward appearance dataset.

Dange and Yengantiwar in 2013 built up an eye estimation method by a machine vision-based idea. A little monochrome camera for security was utilized that directly concentrating on the driver's face which scans the driver's eyes to distinguish tiredness. This paper portrays the condition of eyes whether they are open or close dependent on the Viola Jones calculation.

B.N. Manu in 2016 portrayed a strategy for detecting the drowsiness by three stages. These stages were utilized for facial highlights recognition with the use of Viola Jones, the eyes tracking

Figure 1. This figure shows the risk aspects of fatigue (source: https://www.interdynamics.com/wpcontent/uploads/2015/07/ RiskBasedApproachToManagingFatigue.png)

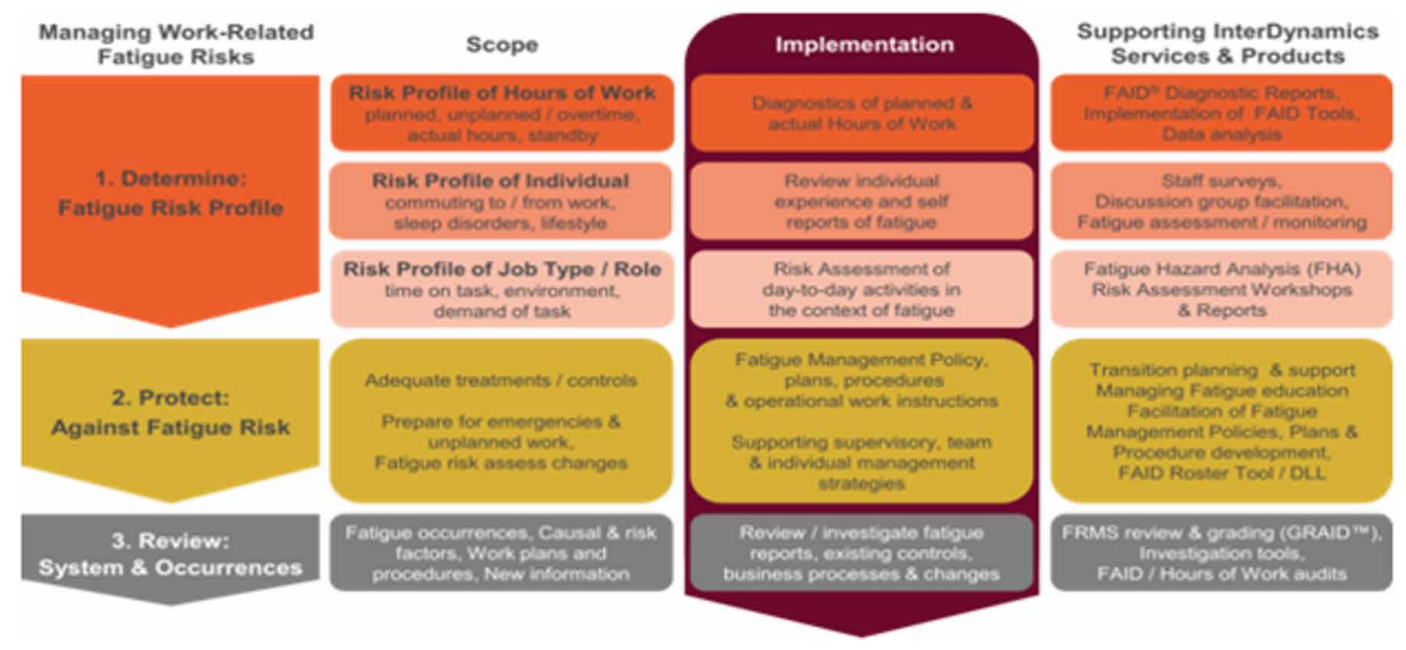




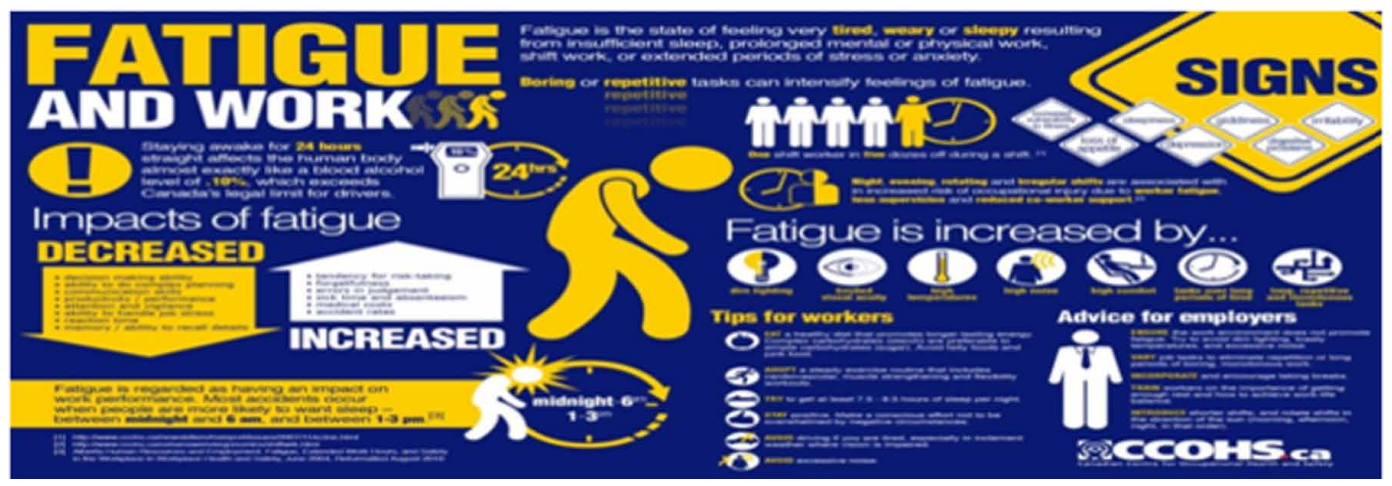

and yawning identification. In order to classify the consecutive frames into two states, a binary linear support vector machine classifier was first fatigue and another non-fatigue state and then the alarm sound was formed if it was above the threshold time.

Wang et al. in 2018 proposed a framework for recognizing the eyes state with the deep learning approach, which shows collection of the data that improves quality of anti-fake and finally results in the promotion of performance of biometric identification.

Page and Ashlock in 2013 included previous work modeling stress in the workplace. The use of agent training was done by binary automata to adopt the successful behavior of highly productive mentors. This paper was only based on the Stress that is accumulated through long working hours which affects the performance of the agent by decreasing productivity.

Singh, Singh, and Singh in 2016proposed a new approach for eyes extraction from frontal facial pictures. Sobel edge operator and morphological operation was used by them for extraction of the eyes.

Salmam, Madani, and Kissi in 2016 included emotion recognition from facial expressions in three steps: face detection, features extraction and classification of expressions on the bases of two databases JAFEE and COHEN.

Hajare and Dule in 2011 presented different techniques of modeling the mood and also fatigue in any individual. Lots of parameter like facial expression, tone of voice that is speech synthesis, posture, gesture etc. was used for mood detection and fatigue detection of human.

Hajare and Dule in 2012 presented the effect of fatigue on the lives of workers. When employees / workers were tired, they were not able to work fast. Due to fatigue, it is possible to increase the risk of fatigue on the site because fatigued employees are less able to respond actively in several circumstances.

Kashyap and Sharma in 2017 proposed a new methodology of detecting the fatigue level of the driver using the MATLAB tool. Drowsiness Detection System has been developed with the use machine vision-based concepts. The framework utilizes a little camera that straight forwardly centered around driver's face and screens the driver's eyes to recognize exhaustion or tiredness.

\section{LITERATURE SURVEY}

\section{Techniques used for Fatigue Detection}

\section{Cognition and Mapped Binary Patterns}

Facial recognition framework comprises of face identification, extraction of features and furthermore separating the expressions into various classes. This strategy incorporates LBP (Local Binary 
Patterns) in which LBP operator calculates the dim scale model between every pixel of the picture and furthermore its nearby paired neighbors. At that point after this it encodes the binary succession of picture for the formation of local binary patterns (Qi, Li, Wang, Zhang, Xing, Gao, \& Zhang, 2018).

\section{Sobel Edge Detection}

In this method, Sobel operator performs the 2-D spatial estimation on the picture in which each pixel of the picture worked by this operator and further estimates the inclination of the picture for each pixel (Singh, Singh, \& Singh, 2016).

\section{Haar-like features}

This technique is used to remove the background from an image. Viola and Jones algorithm used Haar wavelets for the development of Haar-like features. This feature requires the rectangular area on the detection window. To detect the eyes from the face Haar-like features are used. It is a classifier trained by applying any machine learning (Dange \& Yengantiwar, 2013)

\section{Static Bayesian Network}

The fundamental reason for this procedure is to deduce unobservable events from the observed information. Firstly, it distinguishes hypothesis events and after that grouped them in to a lot of totally unrelated events. Also, it recognizes the observed information that can demonstrate some hypothesis and afterward combines them in to data factors (Ji, Lan, \& Looney, 2006).

\section{Gaze Estimation}

This method is used for pupil's detection of the eye. Gaze estimation produces the desired pupil effects to extracts the dark shade (Ji, Zhu, \& Lan, 2004).

\section{Fuzzy Expert System}

It is a mechanism used to find the fatigue by the facial expressions like movement of eyes and also its angle. It is also to calculate the pupil movement. It measures the collision detected and reduces the cost (Jagadeesh \& Patil, 2017).

\section{Connected Component Analysis}

It consists of groups and clusters in which clusters are put to an aspect ratio test. This method is effective in the background objects. Also, it improves gray-level dilation (Kumar, Raja, \& Ramakrishnan, 2002).

\section{Convolution Neural Network}

It is used to reduce the multiple levels of data and enables complex feature to be learn. Convolution layer is the center structure of $\mathrm{CNN}$ where each layer contains some significant highlights and processes the info include. It combines all possible structure and presents a useful detail within a region (Qi, Li, Wang, Zhang, Xing, Gao, \& Zhang, 2018).

\section{DISCUSSION}

Human fatigue detection becomes very important in software industries. Few methods are discussed here for feature extraction that can be utilized for fatigue detection. The comparisons between different techniques are shown below in the table1.

Here the algorithms are being used for image processing for feature extraction of the human face. This paper proposes the best suited algorithm for human fatigue detection in the software industry.

Cognition and Mapped Binary Patterns (Qi, Li, Wang, Zhang, Xing, Gao, \& Zhang, 2018) is used to detect the facial features such as, eyes, nose, mouth, both cheeks etc. It uses the LBP method 
for facial contour extraction. The advantage of this algorithm is that its computational efficiency is very high but automatic face localization is not possible by this algorithm. The second algorithm is Sobel Edge Detection (Singh, Singh, \& Singh, 2016) which extracts the facial region features and then eyes features. In this algorithm measurement of eyes is done by measuring the distance within the centroid of eyes and angle between the centroids of eyes. The main advantage of this algorithm is that it can detect edges and their motion and also it reduces complexity but it cannot results in accurate edge detection. The third algorithm is Haar-like features (Dange \& Yengantiwar, 2013) that extract the eyes features and it removes image background Osan image with region of face as output. The advantage of this algorithm is very important for fatigue detection in software industries for the developers and the coders. As the results are more accurate even if image is affected by illumination of computer screen. This algorithm is only one which is not affected by the factor of illumination, but response time is high. The fourth algorithm is Static Bayesian Network (Ji, Lan, \& Looney, 2006) it extracts the features like eye movement, head movement, facial expressions, heart activities etc. This algorithm distinguishes speculation occasions and gatherings them into a lot of fundamentally unrelated occasions. It reduces complexity but it is computationally infeasible. The fifth algorithm is Gaze Estimation (Ji, Zhu, \& Lan, 2004) which extracts the facial features like nasal root, nose tip, lower face, right eye, left eye. This algorithm includes three parts: detection of pupil, tracking, gaze estimation and its mapping and have advantage of best accuracy in results but it affected by the accurate calibration procedure. The sixth algorithm is Fuzzy Expert System (Jagadeesh \& Patil, 2017) extracts features like yawning and eye closure for calculation of internal pupil angle and movement. It gives fast response to the problem, but it lacks in Meta knowledge. The seventh algorithm is Connected Component Analysis (Kumar, Raja, \& Ramakrishnan, 2002) which extracts eyes features. It only extracts only those pair of clusters that lie within the angle of line joining the centroid of one cluster. The main advantage is that it allows local and global information are taken into account for segmentation but it is Semi-automatic. The last algorithm is Convolution Neural Network (Qi, Li, Wang, Zhang, Xing, Gao, \& Zhang, 2018) which extracts features like blinking, eye closure duration, and yawning etc. This algorithm calculates the eye closure percentage over time. It is having high accuracy in the results, but it has a high computational cost with a need of a lot of training data.

\section{CONCLUSION}

There has been progress in face detection techniques over the past few years. Few techniques for extracting and analysing human fatigue detection features are discussed. By comparing it has been that all the techniques have normal ability like high accuracy, reduce complexity, less time taken etc. But there is only one algorithm that can be used for resolving the issue of fatigue detection of people working in software industries and the issue is the illumination factor. After comparison, it was concluded that illumination of the computer screen is the very important factor in software industries which effects person who is working on the system. So, to resolve this issue an algorithm Haar-like feature can be used for detecting the fatigue of the worker. This paper can be helpful for the researchers who willing to start working of fatigue detection. This paper not only provides a comparative study of the algorithms used for fatigue detection but also helps to understand the use of different facial features, their classification and their use for worker fatigue detection. 
Table 1. Comparison between different fatigue detection techniques

\begin{tabular}{|c|c|c|c|c|c|}
\hline S. no. & Techniques & Features & Methodology used & Advantages & Disadvantages \\
\hline 1. & $\begin{array}{l}\text { Cognition and Mapped } \\
\text { Binary Patterns }\end{array}$ & $\begin{array}{l}\text { eyes, both cheeks, } \\
\text { nose, mouth these are } \\
\text { the features that being } \\
\text { extracted }\end{array}$ & $\begin{array}{l}\text { Find out facial shapes by } \\
\text { nearby paired example } \\
\text { (LBP) strategy and } \\
\text { after that divides the } \\
\text { facial locale by utilizing } \\
\text { pseudo 3D model }\end{array}$ & $\begin{array}{l}\text { Computational } \\
\text { efficiency is high }\end{array}$ & $\begin{array}{l}\text { Automatic face } \\
\text { localization may not be } \\
\text { completely accurate }\end{array}$ \\
\hline 2. & Sobel Edge Detection & $\begin{array}{l}\text { Extraction of facial } \\
\text { region and then edge } \\
\text { detection of eyes }\end{array}$ & $\begin{array}{l}\text { 2-D spatial gradient } \\
\text { measurement is } \\
\text { performed by Sobel } \\
\text { operator Eye region is } \\
\text { extracted by measuring } \\
\text { the distance from left or } \\
\text { top or base to centroid } \\
\text { and point between the } \\
\text { centroids }\end{array}$ & $\begin{array}{l}\text { Can identify edges and } \\
\text { their orientations } \\
\text { Reduction of } \\
\text { Complexity }\end{array}$ & $\begin{array}{l}\text { Cannot create exact edge } \\
\text { recognition with light } \\
\text { and smooth edge } \\
\text { Time consuming } \\
\text { Complex computation }\end{array}$ \\
\hline 3. & Haar like features & $\begin{array}{l}\text { Detection of face and } \\
\text { then extraction of eye } \\
\text { feature }\end{array}$ & $\begin{array}{l}\text { It is a classifier that } \\
\text { prepared with couple } \\
\text { of positive and negative } \\
\text { pictures } \\
\text { Removal of background } \\
\text { from a picture is done } \\
\text { and only region of face } \\
\text { is extracted as output }\end{array}$ & $\begin{array}{l}\text { Calculation speed is fast } \\
\text { High detection accuracy } \\
\text { It can result more } \\
\text { accurate even if image is } \\
\text { affected by illumination }\end{array}$ & Longer training time \\
\hline 4. & Static Bayesian Network & $\begin{array}{l}\text { Features like eye } \\
\text { movement, head } \\
\text { movement, facial } \\
\text { expressions, heart } \\
\text { activities etc }\end{array}$ & $\begin{array}{l}\text { Recognize hypothesis } \\
\text { events and gathering } \\
\text { them into a lot of } \\
\text { fundamentally unrelated } \\
\text { events } \\
\text { Recognize related } \\
\text { information that uncover } \\
\text { something about } \\
\text { hypothesis variable }\end{array}$ & Reduces complexity & $\begin{array}{l}\text { Computationally } \\
\text { infeasible } \\
\text { Un automatic }\end{array}$ \\
\hline 5. & Gaze Estimation & $\begin{array}{l}\text { Feature extraction like } \\
\text { nasal root, nose tip, } \\
\text { lower face, right eye, } \\
\text { left eye }\end{array}$ & $\begin{array}{l}\text { It incorporates } \\
\text { three sections: pupil } \\
\text { recognition, tracking, } \\
\text { calibration of gaze and } \\
\text { its mapping It shows } \\
\text { the result with } 96 \% \\
\text { accuracy }\end{array}$ & $\begin{array}{l}\text { As the quantity of } \\
\text { alignment focuses } \\
\text { builds, the tracking of } \\
\text { gaze turns out to be } \\
\text { progressively exact }\end{array}$ & $\begin{array}{l}\text { The tracking of gaze is } \\
\text { influenced by the exact } \\
\text { adjustment methodology }\end{array}$ \\
\hline 6. & Fuzzy Expert System & $\begin{array}{l}\text { Feature like yawning } \\
\text { and eye closure }\end{array}$ & $\begin{array}{l}\text { It investigates the } \\
\text { fatigue by observing } \\
\text { the yawn and eye state } \\
\text { and for computation of } \\
\text { interior pupil point and } \\
\text { movement }\end{array}$ & $\begin{array}{l}\text { Fast response } \\
\text { Cheaper }\end{array}$ & Lack of meta-knowledge \\
\hline 7. & $\begin{array}{l}\text { Connected Component } \\
\text { Analysis }\end{array}$ & Extraction of eye areas & $\begin{array}{l}\text { Only that pair of clusters } \\
\text { are chosen that lie } \\
\text { within the angle of line } \\
\text { joining the centroid of } \\
\text { one cluster }\end{array}$ & $\begin{array}{l}\text { Allow local and } \\
\text { global information are } \\
\text { taken into account for } \\
\text { segmentation }\end{array}$ & $\begin{array}{l}\text { Semi-automatic: } \\
\text { requires user input and } \\
\text { expertise }\end{array}$ \\
\hline 8. & $\begin{array}{l}\text { Convolution Neural } \\
\text { Network }\end{array}$ & $\begin{array}{l}\text { Facial expressions like } \\
\text { blinking, eye closure } \\
\text { duration, yawning }\end{array}$ & $\begin{array}{l}\text { It can extract feature } \\
\text { and have better ability } \\
\text { of characterize It } \\
\text { basically calculates the } \\
\text { eye closure percentage } \\
\text { over time }\end{array}$ & $\begin{array}{l}\text { Accuracy is high in } \\
\text { image recognition } \\
\text { problems }\end{array}$ & $\begin{array}{l}\text { High computational cost } \\
\text { Need a lot of training } \\
\text { data }\end{array}$ \\
\hline
\end{tabular}




\section{REFERENCES}

Bharathi, C. J. (2014). Detection of drowsiness in human eye using SVM. International Journal of Innovative Research in Computer and Communication Engineering, 2(2), 3080-3088.

CCOH. (n.d.). Fatigue [infographic]. Retrieved from https://www.ccohs.ca/images/products/infographics/ download/fatigue.jpg

Chellappa, R.E. (2017). Fatigue Detection Techniques: A Review. International Journal of Pure and Applied Mathematics, 117(16), 503-510.

Chowdhury, R.S., \& Kavakli, M.H. (2018). Sensor Applications and Physiological Features in Drivers' Drowsiness Detection: A Review. International Research Training Program.

Dange, T. Y. (2013). Eye Estimation to detect Drowsiness. Proceedings of the National Conference on Innovative Paradigms in Engineering and technology. Academic Press.

Hajare, C. H. (2012). Analyzing the Biosignal to Make Fatigue Measurement as a Parameter for Mood Detection. International Journal of Computer Science \& Information and Technology, 3(3), 4469-4471.

Hajare, S. D. (2011). A Survey on Mood Condition Detection and Fatigue Measurement Methodology. Proceedings of the $2^{\text {nd }}$ National Conference on Information and Communication Technology (pp. 11-13). Academic Press.

Interdynamics. (2015). Risk based approach to managing fatigue. Retrieved from https://www.interdynamics. com/wpcontent/uploads/2015/07/RiskBasedApproachToManagingFatigue.png

Jagadeesh, N., \& Patil, C. M. (2017, August). Development of a new methodology for iris algorithm in biometric authentication using hamming distance concepts. Proceedings of the 2017 International Conference on Energy, Communication, Data Analytics and Soft Computing (ICECDS) (pp. 3362-3365). IEEE. doi:10.1109/ ICECDS.2017.8390082

Ji, Q., Lan, P., \& Looney, C. (2006). A probabilistic framework for modeling and real-time monitoring human fatigue. IEEE Transactions on Systems, Man, and Cybernetics. Part A, Systems and Humans, 36(5), 862-875.

Ji, Z., Zhu, Z., \& Lan, P. (2004). Real-Time Nonintrusive Monitoring and Prediction of Driver Fatigue. IEEE Transactions on Vehicular Technology, 53(4), 1052-1068. doi:10.1109/TVT.2004.830974

Kai, T. W. C., \& Jian, L. H. (2018). Fatigue reliability analysis and life bench test of buffer block in car damper. Proceedings of the IEEE International Conference on Engineering Technology and Innovation. IEEE Press. doi:10.1109/ICE.2018.8436252

Kashyap, V. S. (2017). Drowsiness detection system using Matlab. International Journal of Advance Research in Science and Engineering, 6(5), 689-695.

Kumar, R. T., Raja, S. K., \& Ramakrishnan, A. G. (2002, September). Eye detection using color cues and projection functions. [). IEEE.]. Proceedings - International Conference on Image Processing, 3, III-III.

Liu, Z. L., \&Wang, Zhao (2010). A practical driver fatigue detection algorithm based on eye state, Proceedings of the Asia Pacific Conference on Postgraduate Research in Microelectronics and Electronics (PrimeAsia). IEEE Press. doi:10.1109/PRIMEASIA.2010.5604919

Manu, B.N. (2016). Facial features monitoring for real time drowsiness detection. Proceedings of the $12^{\text {th }}$ International Conference on Innovations in Information Technology (IIT) (pp. 78-81). Academic Press. doi:10.1109/innovations.2016.7880030

Mohsin, H., \& Abdullah, S. H. (2017). Pupil detection algorithm based on feature extraction for eye gaze. Proceedings of the 2017 6th International Conference on Information and Communication Technology and Accessibility (ICTA). IEEE Press. doi:10.1109/icta.2017.8336048

Page, D. A. (2013). Binary decision automata modeling stress in the workplace. Proceedings of the 2013 IEEE Congress on Evolutionary Computation (pp. 3331-3338). IEEE Press. doi:10.1109/CEC.2013.6557978

Qi, M. L., Li, M., Wang, Q., Zhang, H., Xing, J., Gao, Z., \& Zhang, H. (2018). Facial Expression Recognition Based on Cognition and mapped Binary Patterns. IEEE Access, 6, 18795-18803. doi:10.1109/ACCESS.2018.2816044 
Salmam, F. Z., Madani, A., \& Kissi, M. (2016, March). Facial expression recognition using decision trees. Proceedings of the 2016 13th International Conference on Computer Graphics, Imaging and Visualization $(C G i V)$ (pp. 125-130). IEEE. doi:10.1109/cgiv.2016.33

Singh, M. S., \& Singh (2016). Face detection and eyes extraction using Sobel edge detection and morphological operations. Proceedings of the IEEE Conference on Advances in Signal Processing (CASP) (pp. 295-300). IEEE Press. doi:10.1109/CASP.2016.7746183

Sontakke, M. (2015). Efficient Driver Fatigue Detection and Alerting System. International Journal of Scientific Research Publications, 5(7), 1-4.

Wang, H. W., \& Zhao, J. Y. (2011). Driver Fatigue Detection Technology in Active Safety Systems. Proceedings of the International Conference on Remote Sensing, Environment and Transportation Engineering (pp. 30973100). IEEE Press. doi:10.1109/RSETE.2011.5964969

Wang, Z., Li, C., Shao, H., \& Sun, J. (2018). Eye Recognition With Mixed Convolutional and Residual Network (MiCoRe-Net). IEEE Access, 6, 17905-17912. doi:10.1109/ACCESS.2018.2812208

Wu, H., Yokoyama, T., Pramadihanto, D., \& Yachida, M. (1996, October). Face and facial feature extraction from color image. Proceedings of the Second International Conference on Automatic Face and Gesture Recognition (pp. 345-350). IEEE. doi:10.1109/AFGR.1996.557289

Zhang, L. G. L., \& Su, Z. X. (2017). Driver Fatigue Detection based on Eye State Recognition. Proceedings of the International Conference on Machine Vision and Information Technology (pp. 105-110). IEEE Press. doi:10.1109/CMVIT.2017.25

Zhao, Y.L. (2011). Human Eye Feature Extraction Based On Segmented Binarization. Proceedings of the IEEE International Conference on Biomedical Engineering and Informatics (pp. 304-307). IEEE Press. doi:10.1109/ BMEI.2011.6098343

Vikram Bali has received his B. Tech (CSE) from REC, Kurukshetra, M.E. (CSE) from NITTTR, Chandigarh and PhD from Banasthali Vidyapith, Rajasthan. He has more than 18 years of rich academic experience. He is a Professor \& Head of Department (CSE) at JSS Academy of Technical Education, Noida. He is life time member of Indian Society for Technical Education (ISTE), Computer Society of India (CSI) and Institution of Engineers (IE). He has contributed 21 research papers in international journal and 7 research papers in national conferences/ proceedings and edited books. He has also attended Faculty Enablement programme organised by Infosys and NASSCOM. He has been the member of board of studies of different Indian universities and is a member of the organizing committee for various national and international seminar/conferences. He has written books on the fundamentals of cyber security and laws, software engineering and operating systems. He is a reviewer for many international journals of repute for Inderscience and IGI Global. His research interest includes software engineering, cyber security, automata theory, CBSS, and ERP. 